\title{
INFLUENCE OF MONTORING: FOG AND EDGE COMPUTING
}

\author{
VIVEK KUMAR PRASAD, MADHURI BHAVSAR AND SUDEEP TANWAR*
}

\begin{abstract}
The evolution of the Internet of Things (IoT) has augmented the necessity for Cloud, edge and fog platforms. The chief benefit of cloud-based schemes is they allow data to be collected from numerous services and sites, which is reachable from any place of the world. The organizations will be benefited by merging the cloud platform with the on-site fog networks and edge devices and as result, this will increase the utilization of the IoT devices and end users too. The network traffic will reduce as data will be distributed and this will also improve the operational efficiency. The impact of monitoring in edge and fog computing can play an important role to efficiently utilize the resources available at these layers. This paper discusses various techniques involved for monitoring for edge and fog computing and its advantages. The paper ends with a case study to demonstarte the need of monitoring in fog and edge in the healthcare system.
\end{abstract}

Key words: IoT, Cloud Computing, Fog Computing, Edge Compuitng, Monitoring.

AMS subject classifications. $68 \mathrm{M} 20,68 \mathrm{M} 14$

1. Introduction. Cloud Computing [1] is an important platform for the industries. The establishments which trust heavily on data are progressively likely to make use of cloud, edge, and fog computing infrastructure. The aforementioned architecture will allow the organizations to take advantages of a variety of computing and data storage resources such as Industrial Internet of things(IIoT) and have their different layers with respect to cloud,fog, and edge [2]. The edge computing allows processing to be done locally at numerous decision points for the determination of dropping the network traffic flow. Fog computing and edge computing seem similar since they together include carrying intellect and processing nearer to the formation of information. However, the difference between the two lies in where the place of intelligence and compute power is positioned. A fog environment homes intelligence at the local area network (LAN). This architecture communicates data from endpoints to a gateway, then this is conveyed to sources for processing and yield transmission. Whereas the edge computing chairs processing power and intelligence in devices like embedded automation controllers.

The Fog and edge computing's main goal are to gather, process and analyze the data from the resources more efficiently as compared to the architecture of the outmoded cloud with similar objectives [3]; which are listed below:

- To decrease the volume of data directed towards the cloud

- To reduce the internet and network latency

- To progress system reply time in isolated mission-critical submissions(tasks).

The key variance between the dual theories is that both fog computing as well as the edge computing include the transfer of processing and intelligence abilities down nearer to where the information instigate; i.e. at the network edge [4]. Hence the main difference between the two constructions is exactly where the computing power and intelligence are kept and has been discussed here.

- Fog computing drives intelligence down to the local area network (LAN) level of network design and architecture, processing data in a fog node ( or the gateway of IoT).

- Edge computing shoves the processing power, intelligence, and communication capabilities of an edge appliance or gateway directly into devices such as PACs (Programmable Automation Controllers).

In both the cases, the data will be generated from the source such as physical assets like sensors, pumps etc These devices achieve a task in the physical world like pumping water or sensing the biosphere around them; which are called as things that results into the (IoTs) Internet of Things.

The cloud is the base for edge and fog computing which has been depicted in Fig 1.1 where the cloud is used as the center for data analytics and business intelligence [5] and below cloud fog will be present and does analysis in the local network and after that, another layer is present which is called edge layer, where the computation is performed on the distributed devices (or nodes) itself, such as edge devices or smart devices as to disparate to mainly taking place in a integrated cloud environment.Even the response time too will change

*CSE Department, Nirma University, Ahmedabad, Gujarat, India (vivek.prasad@nirmauni.ac.in, madhuri.bhavsar@nirmauni.ac.in, sudeep.tanwar@nirmauni.ac.in). 


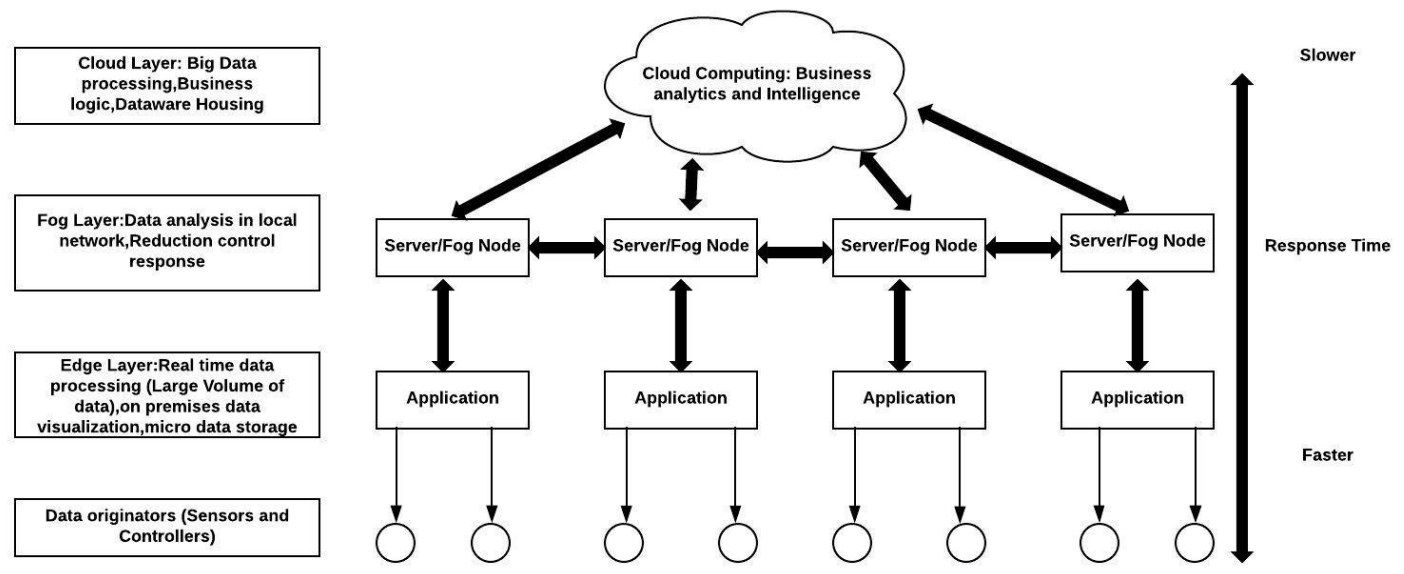

FIG. 1.1. Data processing layer stack in cloud, fog and edge computing

as we move from bottom to top, as the load itself will be distributed. The advent of technologies such as the Internet of Thing(IoT), Mobile edge computing (MEC) [6], software-defined networks [7] and network function virtualization (NFV) [8] are strongly challenging the cloud model for distributing its services among these technologies, even the prognostication regarding the number of connected applications and devices which will munch the cloud resources and will also generate massive volumes of data is a severe challenge for the current model for cloud computing. Hence there is a requirement for the advance of appropriate management systems which will enable an operator to aggregate, give description the availability of the distributed resources and implementing different kinds of services which will be managed by the operators itself. Nevertheless designing and architecting the compatible management system is a challenging task because of the Fog/Edge infrastructure; that is significantly differs from the traditional cloud with respect to dynamicity, heterogeneity and impending enormous distribution of the resources and networking environments. In this paper, the focus is on the various aspects of monitoring the services which are the key elements to any of the management systems of distributed computing environments. Several solutions has been proposed for the cloud, grid and clusters however none of them are well suited for the Fog/Edge Computing and the goal of this paper is to pave the way towards the monitoring systems which are well suited for Fod/Edge computing. The multilayer architecture depicted in Fig. 1.1 offers the following improvements over the classical cloud computing technique are as follow:

- The Network Traffic will reduce: Edge computer layer are able to filter needless data and significantly consolidate only important information that must be flowed into the cloud Computing and then received, processed and stored.

- Applications Performance will Increase: As latency will be reduced because the data processing is happening at the local edge nodes which are placed after to end-devices or end-users instead of the centralized cloud.

- Facilitating load balancing for the approaches used: Scaling the power of computation locally on the edge nodes instead of running entirely on the cloud.

- Minimizing the consumption of energy: The jobs can be offloaded from the end devices to edge nodes instead of cloud which are situated faraway. This will decrease the energy consumption of the end nodes or devices.

The contribution of the paper are listed below:

- To identify the various monitoring requirements in the dynamic environs for edge and fog computing

- The Qualitative study of major solutions which are prevailing

- To gather the influence of the deployment policy of functions for monitoring services for functional and non functional properties of edge and fog computing.

The rest of the article has been organized as follows: section 2 presents the problem statement and the key 


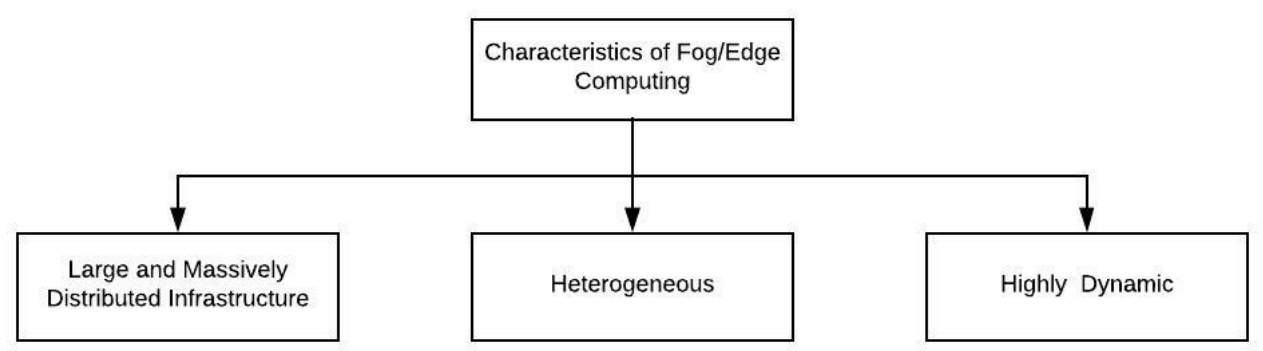

FIG. 2.1. Characteristics of the Fog/Edge Computing

properties of the monitoring services and its associated metrics. Section 3 deliberates the study of the taxonomy of monitoring services in edge and fog computing.Section 4 highlights the perspective of the paper,than in section 5 various open issues has been described.Section 6 describes a case study on healthcare monitoring system, finally followed by the conclusion in section 7. We trust that this paper delivers contributions of interest for the research communal, analyzing the literature and shedding light on the present and forthcoming research matters on fog/edge computing monitoring and prediction.

2. Monitoring Properties and Metrics. As this has been discussed previously that Fog/Edge computing differs from cloud computing with respect to the certain characteristic as shown in Fig. 2.1, such as its distributed infrastructure, heterogeneous and highly dynamicity. These essential features complicate its management [9] and has been discussed below:

- Large and Massively Distributed Infrastructure [10]: The Fog/Edge machinists infrastructure is deployed across manifold sites. There can be distances among these resources and the connections can be in terms of wired (fiber or copper etc.) and wireless (wifi or microwave etc.) links. Here again, the distance and the type of link can affect the latency as well as the bandwidth too.

- Heterogeneous [11]: The Infrastructure is collection of many heterogeneous resources such as

- Server Storage

- Various Routers

- Switches: General Purpose

- Gateways, network links devised

- Computer Servers

- Dynamicity [12]: The tasks which have small lifecycles are often migrated and instantiated. The same is with respect to the Software-defined network capabilities where the modifications of the network will happen and can be changed on the fly. This also happens in the lower level too, where Edge Devices (EDs) may join or leave the network permanently as per the service usages, policies , failures, and operations of the maintenance.

These resources have different characteristics in terms of capacity, usages, and reliability. The virtualization introduces another level of heterogeneity as the operated resources can be physical or virtual.

The monitoring facility on a Fog/Edge [13] with good availability must satisfy the following criteria: The services must be able to oversee a large number of resources. This should hold a instant growth of load or this should able to adapt to an increase of request rate from the perspective of different management systems. The system monitoring can be intended to allow its adaptation to any resource elimination or in the context of virtualized settings where the resources are of fewer consistent. Monitoring services rely on the network to observe the resources available on remote locations, in other words, this should ensure the retransmission of the signals in case of network let-downs. The monitoring levels for fog/edge computing can be classified as:

- Infrastructure or VMs computational resources.

- Dockers Containers

- Networks 
TABLE 2.1

Metrics used for VM Usages

\begin{tabular}{|l|l|}
\hline Goal & Measured metrics (VM-level) \\
\hline Federated clouds Monitoring Mechanism & $\begin{array}{l}\text { Memory,CPU,Network } \\
\text { usage Disk, etc }\end{array}$ \\
\hline Modeling resource usages of cloud tasks & Disk,CPU, Network usage \\
\hline IaaS cloud monitoring & Disk, CPU, Memory \\
\hline IaaS cloud monitoring & Memory, CPU \\
\hline Provisioning Intelligent resource & Memory, CPU \\
\hline
\end{tabular}

TABLE 2.2

Common set of parameters of container table

\begin{tabular}{|l|l|}
\hline Container level metrics & Salient aspects \\
\hline rx_bytes & Bytes identified by the container \\
\hline rx_packets & Packets identified by the container \\
\hline tx_bytes & Bytes transferred by the container \\
\hline tx_packets & Packets transferred by the container \\
\hline Memory_usage & \%memory usage of the container \\
\hline io_service_bytes_write & Bytes written to block device by the container \\
\hline
\end{tabular}

- Measurement of application specific parameters.

Each one of the above has been described below:

- Infrastructure or VMs Computational Resources:VM level monitoring:-

To have well-organized resource utility and avert any issues in monitoring and virtualized resources of the VMs used in the cloud infrastructure and edge nodes are serious issue. Performance optimization can be best attained by efficiently monitoring the utilization of the virtualized resources. Competences for monitoring such resources comprise of resources such as the usage of CPU, memory, storage, and network as shown in Table 2.1 along with their metrics.

- Usage of CPU indicates the quantity of actively used CPU as a fraction of total obtainable CPU in a VM.

- Usage of memory specifies the quantity of memory that is utilized on the designated Infrastructure.

- Disk usage denotes to the quantity of data read or inscribed by a VM. Or this may also designate the percentage of used drive space.

- Network usage is the capacity of traffic on a target network

- In contrast with VMs, the usage of containers which does not involve an Operating System (OS) to boot up to an additional method of server virtualization is swiftly growing in acceptance. An another reason for the same is the container images have a tiny size as compared to VM full images. The example for the container based virtualization platforms such as Container of Google (GKE) and Amazon EC2 container service (ECS) can be used as alternatives to the hypervisor-oriented approach. This is tranquil to pull container images of the application components across the nodes in the cloud. Even the agility is required as the migration technique is the best tool for numerous determinations such as load balancing, reallocating resources (scalability) and dealing with failures due to hardware etc. The the main intention here should be to reduce the downtime in the cloud. Table 2.2 and Table 2.3 indicates the common set of container level policies which can be monitored and useful with respect to the content of the task's adaptation.

- Network

The Network infrastructures [14]conditions tend to change every time and hence the big challenge is to keep the performance of the network high. Even the end to end link quality also depends on the entire communication passing through the edge computing framework regardless of the fact that hat network technologies are being used by the network. The edge paradigm have four forms of connections of the 
TABLE 2.3

Different container level system for monitoring

\begin{tabular}{|l|l|}
\hline Goal & Measured Metrics of container level \\
\hline Time critical tasks:Cloud application & Network Usage,disk,memory,CPU \\
\hline Performance Assessment of virtualization technology & Network Usage,disk,memory,CPU \\
\hline $\begin{array}{l}\text { I/O bound application: Performance Assessment } \\
\text { of virtualization technology }\end{array}$ & Disk \\
\hline Bandwidth - intensive application: support application QoS & Usage of Network \\
\hline
\end{tabular}

TABLE 2.4

Netork:Link quality monitoring systems

\begin{tabular}{|l|l|}
\hline Goal & Network Level Measured Metrics \\
\hline Audio/Video streaming Service: Support Application QoS & Delay \\
\hline Gaming System Cloud: Support Application QoS & Packet Loss,Delay,Throughput \\
\hline Communication Services: Support Application QoS & Throughput, Packet Loss, Delay \\
\hline Multimedia Services: QoE to the users & Jitter, Packet Loss, Delay, Throughput \\
\hline Real time streaming services: Support Application QoS & Jitter, Packet Loss, Delay, Throughput \\
\hline
\end{tabular}

network among the application mechanisms and has been described below

- Communications amongst an edge node and cloud datacentre.

- Communications among any edge nodes itself.

- Communications amongst edge nodes and IoT users/objects.

- Communications amongst/within the cloud data centers.

Table 2.4 indicates the monitoring aspects for the network parameters as well as the metrics.

- The framework for the edge computing is used for any of the undecided dedicated application for any type of software purpose. As every application is running in the cloud requires to be extended to embrace the application level monitoring abilities to measure metrics to identify the current information about the performance and its services status. Table 2.5 summarizes the various works to monitor metrics of application level.

3. Major Monitoring services. Fig. 3.1 indicates the taxonomy for the edge computing monitoring requirements [15] and each branches for the same has been discussed below.

\subsection{Edge Computing framework: Monitoring Requirements.}

1. End to end quality link level [16]

- Identify the end to end network QoS parameters: Certain parameters such as jitter, loss of packet, delay and throughput needs to be monitored for its quality level measurement (QoS) in regard with the end to end network statistics.

- Assist for On-demand network configuration:To manage and control the virtual network resources dynamically the computing framework should provision programmable networks. Which will enhance the monitoring solutions within the edge computing framework.

2. Container level

- Independent from the specific cloud infrastructure provider:The job will be easier if the monitoring solution will be designed for the specific cloud platform, the challenges arise when the generic monitoring solution will be required which may work with multiple providers cloud infrastructure.

- Support dynamic resource management:The monitoring solution will deal with the collection and detection of the dynamic cloud environment. As compared with the centralized data center the edge computing requires agile monitoring approach, as here the end devices are often accessible and unreachable, the mobile devices will be there or they always keep on changing their states.

- Assits for different kind of monitoring hardware visualization: The monitoring solution should be able to make available to the federated clouds in addition to the virtualized hardware. 
TABLE 2.5

Application level monitoring systems

\begin{tabular}{|l|l|}
\hline Goal & Application level measured metrics \\
\hline Twitter:Cloud Application & $\begin{array}{l}\text { Application throughput(data-items/ second), } \\
\text { Response Time(time/ data item) }\end{array}$ \\
\hline SLA violation detection: Cloud Application & Application throughput, response time \\
\hline Web application: Cloud & Application throughput, response time \\
\hline Audio and video services: Cloud Application & Response time \\
\hline Live video streaming services: QoE to the users & $\begin{array}{l}\text { Application throughput,video quality and } \\
\text { dropped frame }\end{array}$ \\
\hline
\end{tabular}

\section{Common terms}

- Muti-tenant cloud environment: This is used for identifying different types of users view and their rights to make use of the systems, for example, the scenarios where the different users will be sharing the same hardware resources and application instances, different users should be able to identify their measurable parameters and gain access to the data which are meant for them only.

- Support desired monitoring time interval:The span of the interval for monitoring is needed to safeguard reliability and avoid overhead in the environment of the systems.

- Assit for long term storage o measured data: The monitoring data should be optimally stored and can be used for the future perspective or deal with future adaptation tactics.

- Support for scalability adaptation policies: The scalable monitoring solution is needed for elastic management of the infrastructure, which also support policies for scaling the monitoring solutions. As these techniques may be useful for autonomic self configuration of the system.

- Assist to setup for automated alerts:The alerts indicate the management aspects of the resources. As an example, the monitoring technique should be able to generate alerts if the operational policies or metrics reaches its threshold value.

- Customized based on monitoring requirements: This is the requirement of every monitoring techniques to changes its policies extension to allow other metrics to be generated/ invoked as and when the conditions of the specific cloud environs change.

4. Application level

- Support application topology and reconfiguration: The monitoring system should be responsive to the different topologies and reconfiguration of the cloud-based environs in order to identify the analytics and operational policies collections for better response of the monitoring scheme.

- Effective measurement of application performance:The small measurement intervals may destructively affect the invasiveness of monitoring tools and on the other aspect, the slow sampling rates will damage the accuracy of monitoring data. Hence the intervals must be determined optimally.

- Support multi tier application:The monitoring system must be able to collect data from multi-tier applications and also should be able to cooperate with the other tier.

- Adaptation to time varying applications:The solutions for the monitoring systems should be adaptable and should change over time ( upgrades), as new definitions of tasks response time matters.

3.2. Fog Computing: Monitoring Requirement. Are summarized in Figure 3.2.

4. Perspectives of the Monitoring Approach. The fog computing [17] is acting like a connexion between the cloud, storage services, and IoT devices, fog computing is a part of cloud computing archetype which makes the cloud closer to the edge network. This delivers a virtualized computational model, networking of the resources and the storage in between clouds datacentre and the end devices. .The properties of fog computing is described in Table 4.1

- Assist to fog nodes configuration:The computational nodes with varied design and formations which are proficient to deliver infrastructure for fog computing at the edge of the network. Such as Vehicular network, cloudlets, servers,base stations and networking devices. These aforementioned environs are 


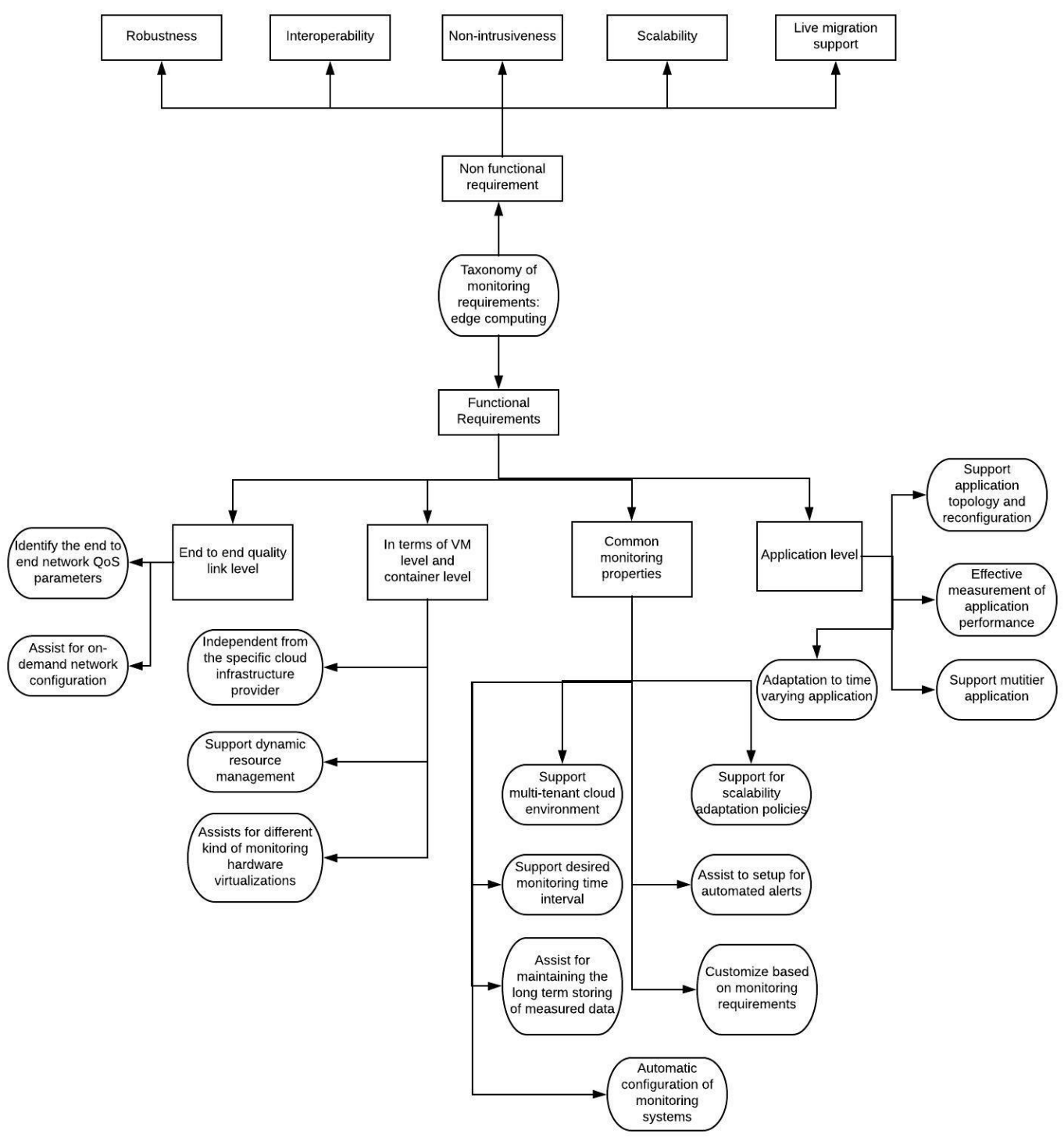

Fig. 3.1. Edge Computing and Monitoring Needs

TABLE 4.1

Properties of fog computing

\begin{tabular}{|l|l|}
\hline Properties & Fog Computing \\
\hline Latency & Less \\
\hline Infrastructure & Limited computing and storage \\
\hline Server nodes location & Edge of the local network \\
\hline Client and server distance & One hop \\
\hline Security Measures & Hard to identify \\
\hline Attack on information & Maximum Probability \\
\hline Deployment & Distributed \\
\hline Awareness about the location & Yes \\
\hline
\end{tabular}




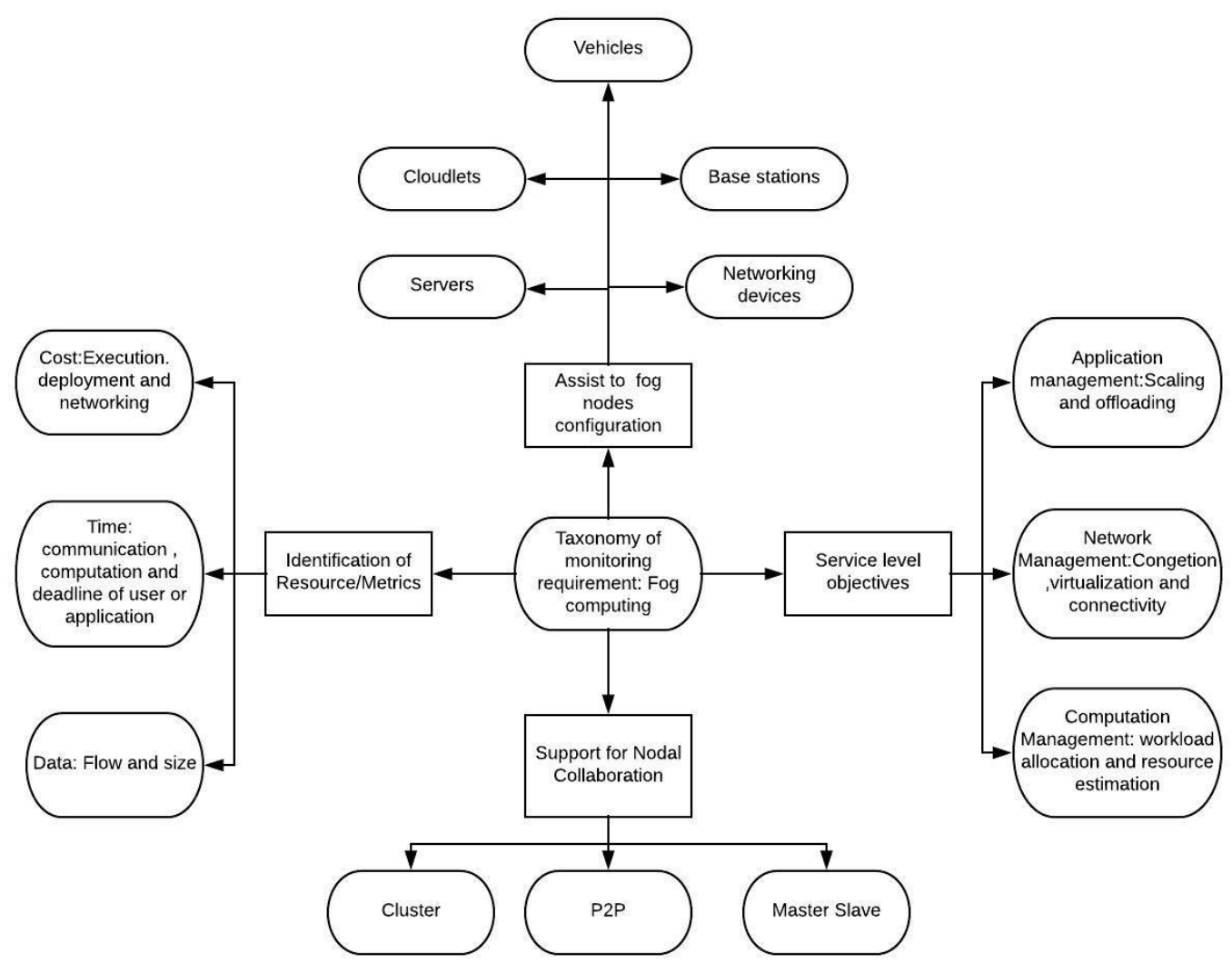

FIG. 3.2. Fog Computing and Monitoring Needs

highly dynamic in nature and monitoring these environs plays an important role for maintaining QoS.

- Service Level objectives:The service level objectives [18] can be reached by developing fog computing as an transitional layer between end devices and cloud data centers and consists of

- Application Management like offloading and scaling.

- Management of network: connectivity,virtualization and congestion

- Computation Management: resource estimation and workload allocation.

Most of the SLOs are administration concerned and cover the issues as latency,resources,cost, power , application and data.

- Support for nodal collaboration:The techniques that are available for nodal collaboration [19] in fog computing has been specified and they are as follow:

- Cluster

- Peer to peer

- Master-slave

The cluster-based association is found to be better in certain cases where the advantage of several Fog nodes work simultaneously. The network overhead plays an important role here.In case of peer to peer(P2P), The access and reliability related issues are prime with respect to P2P nodal association. In master-slave partnership, the master fog node controls processing loads, resource management, control functionalities of the corresponding slave nodes.

- Identification of resources or metrics: Metrics or operational policies can be in terms of

- Cost Execution, deployment and networking.

- Time:communication time,computational time, deadline time etc. 
TABle 4.2

Cloud Monitoring aspects and functional requirements

\begin{tabular}{|l|l|l|l|l|l|l|}
\hline Tool & $\begin{array}{l}\text { Open } \\
\text { Source }\end{array}$ & $\begin{array}{l}\text { Monitoring } \\
\text { of the } \\
\text { container }\end{array}$ & $\begin{array}{l}\text { Quality } \\
\text { Monitoring } \\
\text { of end to } \\
\text { end link }\end{array}$ & $\begin{array}{l}\text { Monitoring } \\
\text { of the } \\
\text { application }\end{array}$ & $\begin{array}{l}\text { Methods for } \\
\text { data storage }\end{array}$ & $\begin{array}{l}\text { GUI } \\
\text { facility }\end{array}$ \\
\hline $\begin{array}{l}\text { Tower } \\
\text { 4Clouds }\end{array}$ & Yes & No & No & Yes & $\begin{array}{l}\text { Graphite, } \\
\text { Influx DB }\end{array}$ & Yes \\
\hline Jcatascopia & Yes & No & No & Yes & $\begin{array}{l}\text { Apache } \\
\text { Cassandra, } \\
\text { MySQL }\end{array}$ & Yes \\
\hline Lattice & Yes & Yes & No & Yes & $\begin{array}{l}\text { Distributed } \\
\text { hash table }\end{array}$ & No \\
\hline DARGOS & Yes & No & No & Yes & $\begin{array}{l}\text { Neutron DBs } \\
\text { and Nova }\end{array}$ & Yes \\
\hline PCMONS & Yes & No & No & No & $\begin{array}{l}\text { MySQL and } \\
\text { Flat file }\end{array}$ & Yes \\
\hline OpenNebula & Yes & No & No & No & $\begin{array}{l}\text { Apache } \\
\text { Cassandra, } \\
\text { MySQL } \\
\text { SQlite }\end{array}$ & Yes \\
\hline Nagios & Yes & No & Yes & No & $\begin{array}{l}\text { MySQL , } \\
\text { Flat file }\end{array}$ & Yes \\
\hline Zabbix & Yes & Yes & Yes & Yes & $\begin{array}{l}\text { SQLite, } \\
\text { MySQL, } \\
\text { Oracle }\end{array}$ & Yes \\
\hline Ganglia & Yes & No & No & Yes & YRDtool & Yes \\
\hline Yenoss & Yes & Yes & $\begin{array}{l}\text { ZODB, } \\
\text { MySQL }\end{array}$ & Yes \\
\hline
\end{tabular}

- Data flow time

In Fog computing model, time is well-thought-out as one of the vital features for service provisioning and effectual resource.

There are various open source monitoring tools that are available for cloud computing and the same can be used to monitor the edge and fog computing environment [20], the same has been depicted in Table 4.2 where these tools are analyzed with the functional requirement (container monitoring, application monitoring, quality monitoring of end to end, methods for data storage and GUI facility) which has been discussed earlier.

5. Suggestions for developing new monitoring approaches . New monitoring approaches can be established in the track of refining the prevailing methods. The new directions for the researchers to develop new methods to the monitoring of edge/fog computing are as described below:

- Management of movement:- The application QoS can decrease and increase rapidly as per the movement of the client device and due to the changing network parameters of the connection between the edge node and the end-users such as bandwidth, delay, and jitter etc. There should be some enhancements to the dynamic service migration methods so that it will be easy to predict the future services(in advance). The nodes should be able to house the growing demand for the service distribution and growing network traffic volume. The edge node should ensure the availability of the services.

- Monitoring data management: For large scale environs, the monitoring investigations will generate massive amount to data collection. Which has to be aggregated, processed and stored afterward.A certain mechanism has to built to reduce these data as they will also consume the bandwidth of the network.

- Decentralization Co-ordination: -The biggest challenge for building decentralized systems is to guar- 


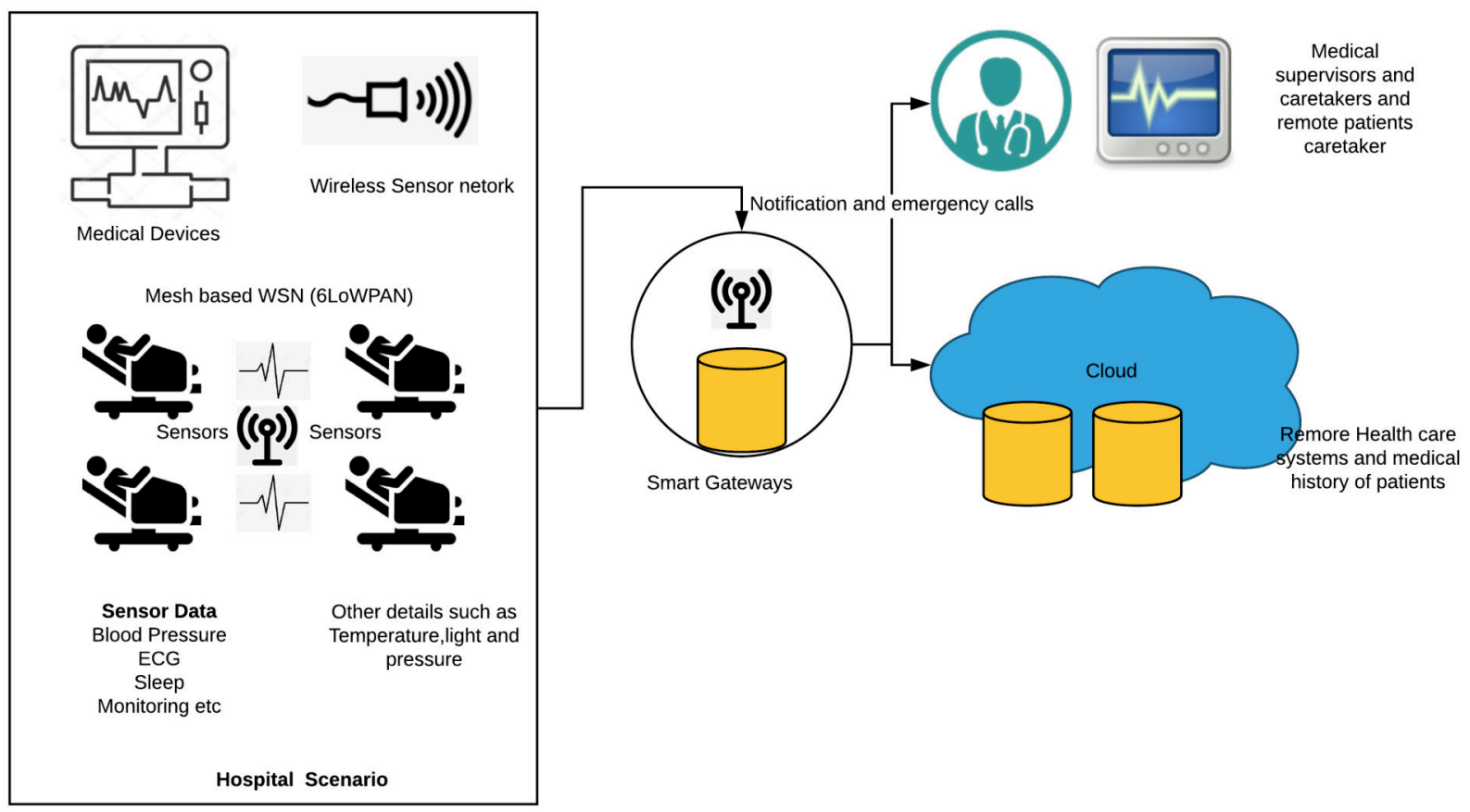

FIG. 6.1. Fog and edge based health monitoring system

antee that dissimilar application components jointly move the whole systems towards to a common approach/goal.

- Peak resource scheduling: the scheduling approach should be intelligent enough to identify the responses upon the uncertainties of the runtime environs as in the case of varying workload, users mobility and network conditions.

- Peak resource scheduling: the scheduling approach should be intelligent enough to identify the responses upon the uncertainties of the runtime environs as in the case of varying workload, users mobility and network conditions.

- Service Replication: With respect the edge/fog computing, the services can be replicated in cloud infrastructure in multi-zone/geographical locations. Replication of the machines has issues such as inconsistencies which need to be handled carefully and when to do replication is an important factor which needs to identify to save time and performance.

The other non functional aspects for monitoring of edge computing are Robustness,interoperability, nonintrusiveness, scalability and live migration suport, used for handling the applications which are running into the corresponding infrastructure of the edge.

6. Case study: Fog and edge computing for IoT based health monitoring systems. Internet of Things is accepted for any organization who wants to make their systems intelligent in real time scenario and its implementation in medical healthcare [21] is proved to be worthy. The architecture for the same has been depicted in Figure 6.1. The gateway is situated at the edge network and this performs the basic necessity function like translating between the protocols. This normally acts as a hub between the cloud service and the ubiquitous devices such as sensor stratum and proved to be performing better in medical healthcare systems [22]. Where this can perform certain important functions such as identifying the location and mobility of the end users. The valuable features can be reinforced in the edge or gateway such as involving intelligence, empowering networking and connect with the adequate power supply. Fog computing in design and architecture of healthcare 
The architecture consists of four components and has been discussed below

- Sensor data related to the medical field and action network: The ubiquitous devices sense and perform the corresponding action by communicating signals from patients body to the control room via the certain usages of the protocols such as 6LowPAN [23], wifi [24], and Zigbee [25].

- Smart e-health network gateway [26]: This results from the different geographically distributed gateways of intelligent gateways, and forming the fog. This receives the data from different networks, usages protocols and provide other higher level of detailed services like reduction in the dimensions, data aggregation, and filtering.

- Systems backend: This includes the platform for cloud computing which implements Dataware housing and analytics of the data collected. These data later can be used for taking important decisions and for medical research epidemiology.

- Features and properties for smart e-health at fog level [27]: In this case, the local pre-processing of the sensors data will be done to do interpretations with respect to medical healthcare and leads to smart e-health gateway.The other important and supportive featues aspects related to the fog are:

- Processing of the local information

- Filtering of the information

- Compression of the information

- Information fusion

- Information Analysis

- Adaptability

- Local storage

- Actuation at the local level

- Security

- Reconfigurability and technical interoperability

- Syntactic and semantic interoperability

7. Conclusion. To simplify the process of self-adaptive and decision-making mechanism in the edge/fog computing environs, monitoring clarifications gather information from diverse levels. In addition to monitoring the virtual resources such as disk, memory, and CPU, it is also important to consider the supplementary levels of monitoring together with the container, network quality, and tasks requirement. The functional and nonfunctional monitoring requirements need to be analyzed for adaptive application for edge/fog computing.The paper also discussed the open challenges for monitoring in edge and fog. The paper ended with the case study of monitoring fog and edge computing with respect to e-health systems and shows that monitoring resources will manage the tasks associated to the health-care data properly or optimally as this includes the various data flow from the processes,data acquisition of sensor nodes and the cloud with the end users.

\section{REFERENCES}

[1] Peter Mell, Tim Grance, et al. The nist definition of cloud computing. 2011.

[2] Lu Tan and Neng Wang. Future internet: The internet of things. In 2010 3rd international conference on advanced computer theory and engineering (ICACTE), volume 5, pages V5-376. IEEE, 2010.

[3] KP Saharan and Anuj Kumar. Fog in comparison to cloud: A survey. International Journal of Computer Applications, 122(3), 2015.

[4] KP Saharan and Anuj Kumar. Fog in comparison to cloud: A survey. International Journal of Computer Applications, 122(3), 2015.

[5] Manas Kumar Sanyal, Biswajit Biswas, Subhranshu Roy, and Sajal Bhadra. A proposed model to integrate business intelligence system in cloud environment to improve business function. In Information Systems Design and Intelligent Applications, pages 282-292. Springer, 2018.

[6] Rodrigo Roman, Javier Lopez, and Masahiro Mambo. Mobile edge computing, fog et al.: A survey and analysis of security threats and challenges. Future Generation Computer Systems, 78:680-698, 2018.

[7] Yang Xu, Marco Cello, I-Chih Wang, Anwar Walid, Gordon Wilfong, Charles H-P Wen, Mario Marchese, and H Jonathan Chao. Dynamic switch migration in distributed software-defined networks to achieve controller load balance. IEEE Journal on Selected Areas in Communications, 37(3):515-529, 2019.

[8] Bo Yi, Xingwei Wang, Keqin Li, Min Huang, et al. A comprehensive survey of network function virtualization. Computer Networks, 133:212-262, 2018. 
[9] Demetris Trihinas, George Pallis, and Marios D Dikaiakos. Admin: Adaptive monitoring dissemination for the internet of things. In IEEE INFOCOM 2017-IEEE conference on computer communications, pages 1-9. IEEE, 2017.

[10] Adrien Lebre, Jonathan Pastor, Discovery Consortium, et al. The DISCOVERY Initiative-Overcoming Major Limitations of Traditional Server-Centric Clouds by Operating Massively Distributed IaaS Facilities. PhD thesis, Inria, 2015.

[11] Ion-Dorinel Filip, Florin Pop, Cristina Serbanescu, and Chang Choi. Microservices scheduling model over heterogeneous cloud-edge environments as support for iot applications. IEEE Internet of Things Journal, 5(4):2672-2681, 2018.

[12] Seong-Min Kim, Hoan-Suk Choi, and Woo-Seop Rhee. Iot home gateway for auto-configuration and management of mqtt devices. In 2015 IEEE Conference on Wireless Sensors (ICWiSe), pages 12-17. IEEE, 2015.

[13] Shanhe Yi, Cheng Li, and Qun Li. A survey of fog computing: concepts, applications and issues. In Proceedings of the 2015 workshop on mobile big data, pages 37-42. ACM, 2015.

[14] Rodrigo Roman, Javier Lopez, and Masahiro Mambo. Mobile edge computing, fog et al.: A survey and analysis of security threats and challenges. Future Generation Computer Systems, 78:680-698, 2018.

[15] Salman Taherizadeh, Andrew C Jones, Ian Taylor, Zhiming Zhao, and Vlado Stankovski. Monitoring self-adaptive applications within edge computing frameworks: A state-of-the-art review. Journal of Systems and Software, 136:19-38, 2018.

[16] Hakiri Akram and Aniruddha Gokhale. Rethinking the design of lr-wpan iot systems with software-defined networking. In 2016 International Conference on Distributed Computing in Sensor Systems (DCOSS), pages 238-243. IEEE, 2016.

[17] Dazhong Wu, Shaopeng Liu, Li Zhang, Janis Terpenny, Robert X Gao, Thomas Kurfess, and Judith A Guzzo. A fog computing-based framework for process monitoring and prognosis in cyber-manufacturing. Journal of Manufacturing Systems, 43:25-34, 2017.

[18] HAN Kui-kui, XIE Zai-peng, and LV Xin. Fog computing task scheduling strategy based on improved genetic algorithm. Computer Science, (4):22, 2018.

[19] Redowan Mahmud, Ramamohanarao Kotagiri, and Rajkumar Buyya. Fog computing: A taxonomy, survey and future directions. In Internet of everything, pages 103-130. Springer, 2018.

[20] Salman Taherizadeh, Andrew C Jones, Ian Taylor, Zhiming Zhao, and Vlado Stankovski. Monitoring self-adaptive applications within edge computing frameworks: A state-of-the-art review. Journal of Systems and Software, 136:19-38, 2018.

[21] Amir M Rahmani, Tuan Nguyen Gia, Behailu Negash, Arman Anzanpour, Iman Azimi, Mingzhe Jiang, and Pasi Liljeberg. Exploiting smart e-health gateways at the edge of healthcare internet-of-things: A fog computing approach. Future Generation Computer Systems, 78:641-658, 2018.

[22] Ammar Awad Mutlag, Mohd Khanapi Abd Ghani, N Arunkumar, Mazin Abed Mohamed, and Othman Mohd. Enabling technologies for fog computing in healthcare iot systems. Future Generation Computer Systems, 90:62-78, 2019.

[23] Tuan Nguyen Gia, Imed Ben Dhaou, Mai Ali, Amir M Rahmani, Tomi Westerlund, Pasi Liljeberg, and Hannu Tenhunen. Energy efficient fog-assisted iot system for monitoring diabetic patients with cardiovascular disease. Future Generation Computer Systems, 93:198-211, 2019.

[24] Chien-Ming Li, Yueh-Ren Ho, Wei-Ling Chen, Chia-Hung Lin, Ming-Yu Chen, and Yong-Zhi Chen. Nasogastric tube dislodgment detection in rehabilitation patients based on fog computing with warning sensors and fuzzy petri net. Sensors and Materials, 31(1):117-130, 2019.

[25] Tuan Nguyen Gia, Imed Ben Dhaou, Mai Ali, Amir M Rahmani, Tomi Westerlund, Pasi Liljeberg, and Hannu Tenhunen. Energy efficient fog-assisted iot system for monitoring diabetic patients with cardiovascular disease. Future Generation Computer Systems, 93:198-211, 2019.

[26] Amir-Mohammad Rahmani, Nanda Kumar Thanigaivelan, Tuan Nguyen Gia, Jose Granados, Behailu Negash, Pasi Liljeberg, and Hannu Tenhunen. Smart e-health gateway: Bringing intelligence to internet-of-things based ubiquitous healthcare systems. In 2015 12th Annual IEEE Consumer Communications and Networking Conference (CCNC), pages 826-834. IEEE, 2015.

[27] Vladimir Stantchev, Ahmed Barnawi, Sarfaraz Ghulam, Johannes Schubert, and Gerrit Tamm. Smart items, fog and cloud computing as enablers of servitization in healthcare. Sensors E Transducers, 185(2):121, 2015.

Edited by: Anand Nayyar

Received: Mar 16, 2019

Accepted: Apr 25, 2019 